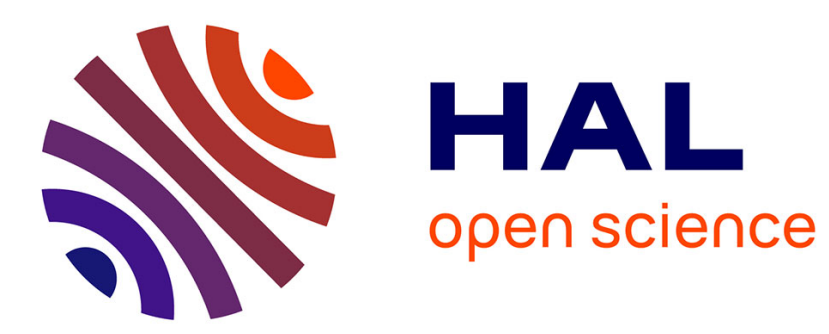

\title{
Coverage Optimization with Connectivity Preservation for UAV Swarms Applying Chaotic Dynamics
}

Martin Rosalie, Matthias R Brust, Grégoire Danoy, Serge Chaumette, Pascal Bouvry

\section{- To cite this version:}

Martin Rosalie, Matthias R Brust, Grégoire Danoy, Serge Chaumette, Pascal Bouvry. Coverage Optimization with Connectivity Preservation for UAV Swarms Applying Chaotic Dynamics. 2017 IEEE International Conference on Autonomic Computing (ICAC), Jul 2017, Columbus, United States. pp.113-118, 10.1109/ICAC.2017.26 . hal-01574636

\section{HAL Id: hal-01574636 https://hal.science/hal-01574636}

Submitted on 16 Aug 2017

HAL is a multi-disciplinary open access archive for the deposit and dissemination of scientific research documents, whether they are published or not. The documents may come from teaching and research institutions in France or abroad, or from public or private research centers.
L'archive ouverte pluridisciplinaire HAL, est destinée au dépôt et à la diffusion de documents scientifiques de niveau recherche, publiés ou non, émanant des établissements d'enseignement et de recherche français ou étrangers, des laboratoires publics ou privés. 


\title{
Coverage Optimization with Connectivity Preservation for UAV Swarms applying Chaotic Dynamics
}

\author{
Martin Rosalie*, Matthias R. Brust*, Grégoire Danoy ${ }^{\dagger}$, Serge Chaumette ${ }^{\ddagger}$ and Pascal Bouvry ${ }^{\dagger}$ \\ ${ }^{*}$ SnT, University of Luxembourg, Luxembourg \\ ${ }^{\dagger}$ SnT/FSTC-CSC, University of Luxembourg, Luxembourg \\ ‡Univ. Bordeaux, LaBRI, UMR5800, Talence, France
}

\begin{abstract}
Cooperative usage of multiple UAVs as a swarm can deliver high-quality surveillance performance. However, the communication capabilities within the UAV swarm must be maintained for local data propagation to swarm members in favor of achieving an efficient global behavior. In this paper, we address the problem of optimizing two adversary criteria for such a UAV swarm: (a) maximizing the area coverage, while (b) preserving network connectivity. Our approach, called $\mathrm{CACOC}^{2}$, solves the problem with a novel chaotic ant colony optimization approach, which combines an Ant Colony Optimization approach (ACO) with a chaotic dynamical system. CACOC ${ }^{2}$ employs swarming behavior to obtain UAV clustering that result in maximized area coverage and preserved network connectivity. We show by extensive simulations how the size of the UAV swarm influences the coverage and connectivity. A metrics comparison chart shows the correlation of coverage and connectivity metrics.
\end{abstract}

Index Terms-cooperative UAV, multilevel swarm, mobility model, ant colony optimization, chaotic dynamics

\section{INTRODUCTION}

Advances in UAV technologies allow the development of new applications for both civilian and military domains. Highend UAVs are delivered with sophisticated on-board systems, extended flight autonomy, increased computing power, ad hoc networking capabilities, and with long-lasting batteries [1]. These improvements make UAVs capable of being deployed as a flying ad hoc network potentially forming a cooperative swarm of UAVs [2], [3]. Advantages of a UAV swarm are for instance that the number of participating UAVs can be adapted as needed and the swarm behavior ensures the resilience of the swarm's communication network in case of hardware failure or harsh weather conditions.

A considerable amount of UAV swarm research focuses on how to deliver high surveillance performance while preserving resilient communication capabilities within the swarm [4], [5], [6]. This coverage optimization problem with connectivity preservation is tremendously important for UAV swarms because the coverage performance highly depends on the overall connectivity dynamics in the swarm. For instance, coverage area coordination and intruder detection notification can only be effectively executed if UAV connectivity is warranted. However, there is a trade-off between coverage and connectivity. For high coverage, the UAVs should intelligently explore the area, while a limited transmission range requires the UAVs to stay in each other's proximity. Therefore, the potential for the UAV swarm to obtain the maximum coverage performance decreases considerably when considering the connectivity requirements. With the UAVs capacity to move freely in the three-dimensional space, an often applied technique on how to obtain superior results for this coverage-connectivity problem is the design of proper mobility models that aim to maximize both criteria (e.g., [4]). In this work, we propose CACOC $^{2}$ (Chaotic Ant Colony Optimization for Coverage with Connectivity), which extends the single criteria Chaotic Ant Colony Optimization for Coverage (CACOC) [3] with a swarming behavior to maintain more stable UAV clusters to deliver connectivity resilience within the swarm.

This work is organized as follows. In Section II, we report on related work and in Section III, we describe the original CACOC UAV mobility model and the novel extension for coverage and connectivity optimization, CACOC ${ }^{2}$. Section IV, provides the experimental setup and settings as well as a description of the metrics used. In Section V we report on the results obtained in the experiments, and we finally conclude our work in Section VI.

\section{RELATED WORK}

Area coverage optimization with connectivity preservation is a tremendously important problem because of its relevance in real-world applications. A commonly used approach to reach superior results for this problem is the design of mobility models that are concerned with coverage and connectivity.

Yanmaz [6] proposes a connectivity- and coverageoptimized mobility model and measures the trade-off between connectivity and spatial coverage. The measure spatial coverage (areal coverage) is defined as the percentage of the sensed target area in a given amount of time, and connectivity is defined as the percentage time the UAVs are connected to a sink averaged for all UAVs.

Yanmaz et al. [9] study single-hop and multi-hop scenarios. Results show that if the network density is high enough, the coverage optimization has less impact on the good performance. However, if the network density is low, a combination of connectivity optimization and coverage optimization is advantageous. 
TABLE I

MOBILITY MODELS PROPOSED FOR THE UAV COVERAGE-CONNECTIVITY PROBLEM.

\begin{tabular}{ll}
\hline Mobility model & Reference \\
\hline Pheromone and clustering based connectivity & Danoy et al, Rosalie et al. [4], [3] \\
Virtual forces-based approach & Brust et al., Yanmaz, Messous et al. [6], [2], [5] \\
First-order flight model & Hauert et al. [1], [7] \\
Belief-based mobility model & Yanmaz et al. [8] \\
Heuristic-based mobility model & Messous et al. [5] \\
Pheromone and forces-based approach & CACOC $^{2}$ (our approach) \\
\hline
\end{tabular}

Yanmaz et al. [8] are interested in UAV movements such that they cover the entire area within a given time period. The introduced belief-based approach uses local information to execute its actions.

Hauert et al. [1], [7] propose a purely communication-based approach to swarming. The authors use artificial evolution to design the mobility and mission model. A second approach deals with army-ant pheromone trails during foraging to be applied to the UAV swarm for the deployment, maintenance and, retraction of communication networks. The main objective is to cover and maintain connectivity between the base and the user.

Brust et al. [2] propose VBCA, a virtual forces clustering algorithm, which imitates the VSEPR model from molecular geometry for the arrangement of UAVs in a clustered swarm. The UAV's position is determined by the distance and role of its neighboring UAVs. VBCA assigns the role of a clusterhead to one UAV. This central UAV acts as a connector influencing the entire topology of the network geometry while individual UAVs are only affected by their direct neighbors. VBCA is maximizing the volume coverage, while maintaining advanced connectivity within the clustered UAV swarm. Most approaches described in this section focus on areal coverage from a plane, i.e. UAVs are flying at a similar altitude. As an exception, VBCA [2] deals with a volume coverage approach.

Messous et al. [5] approach focus on the coverageconnectivity problem with a base station and additional energy constraints (alpha-based mobility model). The decision about the next movement takes into account communication constraints and remaining energy.

The decision making is based on value table where the number of neighbors, energy level and the hop count to the base station determine the next action.

In previous work we address the coverage problem of an area for a swarm of UAVs [3]. Rosalie et al. [3] propose to combine Ant Colony Optimization with chaotic dynamics to improve the coverage of a given area. However, in the described approach connectivity constraints are not considered. Table I summarizes the mobility models used as a method to optimize solutions for the UAV coverageconnectivity problem. We also classified $\mathrm{CACOC}^{2}$ according to Table I to illustrate the fundamental differences of our approach to existing ones.

\section{Chaotic Mobility Models}

In this section, we first briefly summarize CACOC before describing $\mathrm{CACOC}^{2}$, which includes our extension to include connectivity constraints into the coverage optimization approach from CACOC. Both mobility models rely on Ant Colony Optimization (ACO) [10] which aim at imitating the behavior of ants communicating in their colony with pheromones to accomplish complex tasks collaboratively. Ants deposit these pheromones to guide and inform the other members of the colony, this indirect communication process is also referred to as stigmergy. Formal abstractions of this model are used to solve a diversity of optimization problems [10]. Since the pheromones are used to improve a solution, random initial solutions are used to initiate the optimization process. The main idea of CACOC [3] is to take benefit from the structured chaotic dynamics instead of the traditionally used random process. To do so, the CROMM (Chaotic Rössler Mobility Model) is introduced [3] as a fully deterministic mobility model dedicated replacing the random part of an ant colony algorithm.

The CROMM, CACOC and $\mathrm{CACOC}^{2}$ mobility models are described in more details hereinafter. In all three models a UAV moves with a constant speed and starts to choose its direction at each step (left, ahead or right).

\section{A. CROMM}

The dynamical Rössler system [11] is a well-known Ordinary Differential Equations (ODE) system

$$
\left\{\begin{array}{l}
\dot{x}=-y-z \\
\dot{y}=x+a y \\
\dot{z}=b+z(x-c)
\end{array}\right.
$$

producing chaotic dynamics: the solution is a chaotic attractor (Fig. 1 of [3]). From this attractor, we can extract a map (Fig. 1) describing the dynamical properties of the system (for details on the Rössler attractor, the Poincaré section used and the first return map properties see [12]). In CROMM, the first return map gives the direction of the each UAV:

- if $\rho_{n}<1 / 3$ then direction is right;

- if $1 / 3<\rho_{n}<2 / 3$ then direction is left;

- else the direction is ahead.

Such a partition provides a reasonable pattern for exploration [3] including straight forward moves and large circular patterns (see Fig. 1 for period 1 (AAA) and 2 (RARARA) orbits). 
To initialize the system, we choose a different initial condition for each UAV to solve the Rössler system. Giving nonequal initial conditions leads to the same attractor as a solution and to the same first return map. However, the points constituting the return maps are not the same in terms of order of appearance.

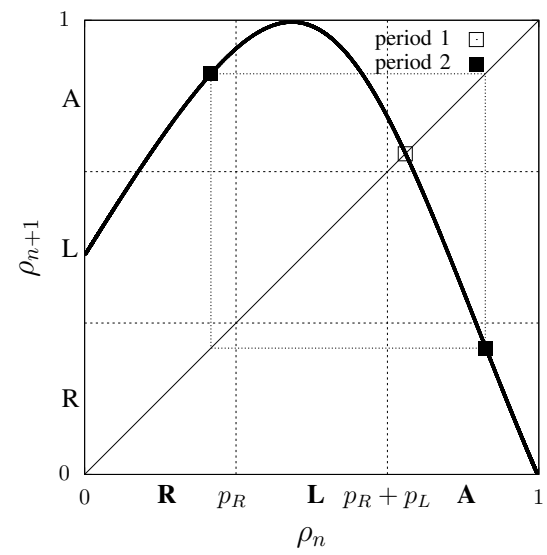

Fig. 1. First return map of the Rössler attractor. This map indicates the UAV directions: L (left), A (ahead) and R (right).

\section{B. $C A C O C$}

When there is no pheromone to guide the UAV, CACOC uses CROMM to obtain the direction of the UAVs. This permits to explore non-visited area. Otherwise, if there are some pheromones, the UAV chooses its next direction according to the total amount of pheromones sensed around him.

We proposed to use again the chaotic dynamic with the next value in the return map to make this choice. Thus, $p_{L}, p_{A}$ and $p_{R}$ values are a percentage partition inversely proportional to the total amount of pheromones respectively sensed on the left, ahead or right:

- if $\rho_{n}<p_{R}$ then direction is right;

- if $p_{R}<\rho_{n}<p_{R}+p_{L}$ then direction is left;

- else the direction is ahead.

Thus, the return map values are used to choose the next direction for each step of the CACOC model (Fig. 1 describes the choice when there are some pheromones around the UAV). The use of pheromones permits to have a system resilient to UAVs failure or loss. If a UAV is out of the system, it will no more deposit pheromones.

\section{C. $\mathrm{CACOC}^{2}$}

The principle of the "boid" movements has been introduced by Reynolds [13]. Three rules reproduce the behavior of a bird in a swarm: collision avoidance, velocity matching, and flock centering. For $\mathrm{CACOC}^{2}$, we combine this model with the "boids" model [14]. As the UAVs have a constant speed, we will not use the velocity matching rule. The purpose of CACOC is to cover a wide area where the UAVs use repulsive pheromones to explore non-visited areas. We then decide to consider that CACOC is another way to implement the first rule of collision avoidance because the pheromones deposit prevents UAVs to move towards the same direction. Thus, we add the third rule of flock centering to force the UAVs to maintain connectivity. As the "boid" model operates with forces, our model only has two forces:

- $\vec{f}_{\mathrm{CACOC}}$ is a vector for the CACOC force giving a direction ( $\mathrm{L}, \mathrm{R}$ or $\mathrm{A})$ that is translated as a vector;

- $\vec{f}_{\text {flock }}$ is a vector for the flock centering force computed with the average value of the last vector used for the other UAVs close to the UAV considered.

We then add these two forces and normalize the obtained vector to have the vector $\vec{d}$ with the constant speed $s$ :

$$
\vec{d}=s \times \frac{\vec{f}_{\text {CACOC }}+f \times \vec{f}_{\text {flock }}}{\left\|\vec{f}_{\text {CACOC }}+f \times \vec{f}_{\text {flock }}\right\|^{2}} .
$$

In this equation $f$ is a parameter to evaluate the influence of the flocking force on the $\mathrm{CACOC}^{2}$ model. We plan to variate this force $f$ from 0.1 to 0.6 by a 0.1 step to calibrate our force model. The purpose is also to evaluate how this connectivity force influences the performance of $\mathrm{CACOC}^{2}$.

\section{EXPERIMENTS}

In this section, we describe the experimental setup and explain the selection of the comprehensive set of metrics. For the experiments, we have analyzed the parameters of the simulations described in Section II and crystallized the most common settings used to be chosen for our experiments. For instance, Yanmaz [6] uses between 1 and 20 UAVs, while the chosen transmission ranges are $68 \mathrm{~m}, 126 \mathrm{~m}, 279 \mathrm{~m}, 500 \mathrm{~m}$, $1000 \mathrm{~m}, 1500 \mathrm{~m}$, and $2000 \mathrm{~m}$ with an area length of $4000 \mathrm{~m}$. In comparison Yanmaz et al. [8] use between 1 to 40 UAVs, with a fixed transmission range of $500 \mathrm{~m}$ and squared simulation area with length $4000 \mathrm{~m}$. Messous et al. [5] vary the UAVs from $5,10,15,20,30$, while the transmission range is $400 \mathrm{~m}$ and the environment is a $4000 \mathrm{~m} \times 4000 \mathrm{~m}$ square area.

\section{A. Experimental setup}

The simulation area is a $4000 \mathrm{~m} \times 4000 \mathrm{~m}$ square, divided into square cells of $10 \mathrm{~m} \times 10 \mathrm{~m}$. The UAVs have a constant speed of $10 \mathrm{~m} / \mathrm{s}$ and all depart from the base station located in the middle of the bottom edge of the map, i.e., position (200, 0 ). At each simulation step, each UAV can conduct one of the following three actions: (1) ahead: the UAV keeps the same

TABLE II

MAIN EXPERIMENTAL PARAMETERS.

\begin{tabular}{ll}
\hline Parameter Name & Parameter Value \\
\hline $\begin{array}{l}\text { Simulation area } \\
\text { Geographical Area }\end{array}$ & $\begin{array}{l}4000 \mathrm{~m} \times 4000 \mathrm{~m} \\
\text { Number of cells }\end{array}$ \\
$\begin{array}{l}\text { UAV Autopilot } \\
\text { UAVs speed }\end{array}$ & $\begin{array}{l}10 \mathrm{~m} / \mathrm{s} \\
\text { ahead, } 45^{\circ} \text { left, } 45^{\circ} \text { right } \\
\text { Possible UAV actions } \\
\text { Initial UAVs position } \\
\text { Experiments }\end{array}$ \\
$\begin{array}{l}\text { Number of UAVs } \\
\text { Force value }(f)\end{array}$ & {$[10,20]$} \\
Mobility Models & {$[0.1,0.2,0.3,0.4,0.5,0.6]$} \\
Simulation steps & 4000 \\
Independent runs & 30 \\
\hline
\end{tabular}




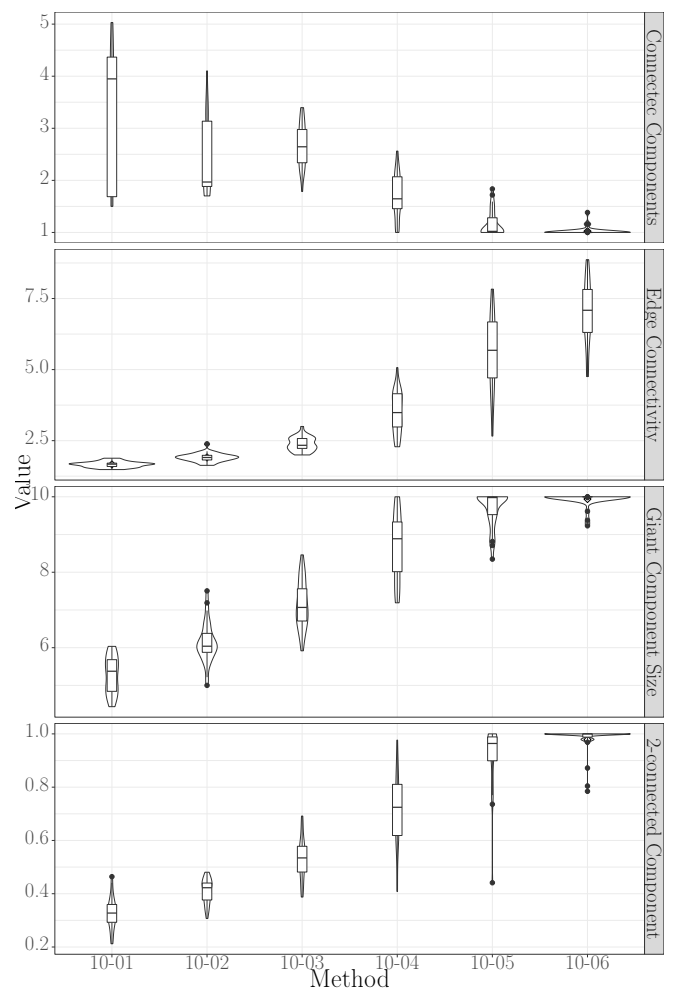

Fig. 2. Connectivity metrics for 10 UAVs.

direction, (2) left: the UAV turns left with a $45^{\circ}$ angle, and (3) right: the UAV turns right with $-45^{\circ}$ angle. To prevent collisions between the UAVs, we assume that each of them has a different flight altitudes [15].

The mobility model with varying parameters has been evaluated with a swarm of 10 and 20 autonomous UAVs equipped with wireless communication capabilities (Tab. II). We ran 30 independent simulations of 4000 steps to obtain significant results.

To evaluate the performance of the different models in terms of area coverage and network connectivity, the following metrics have been used: coverage and recent coverage ratio, fairness, the number of connected components, the size of the giant component, 2-connectivity of the giant component and edge-connectivity. All seven metrics are described in detail in the next section.

\section{B. Metrics}

a) Coverage: The coverage is the portion of the total area visited during the whole simulation. To have a representative value of the coverage, we choose the coverage value at the end of the simulation. It indicates how efficient the models are to visit the total area. We also want to evaluate their initial behavior while there is no guidelines for UAVs. We extract the slope of a linear regression $(a \times t)$ considering the 500 first steps.

b) Fairness of coverage: The fairness of the coverage measures if all cells are regularly and equally scanned. This is computed by the standard deviation of their respective number of scans [16]. To evaluate the fairness during the whole

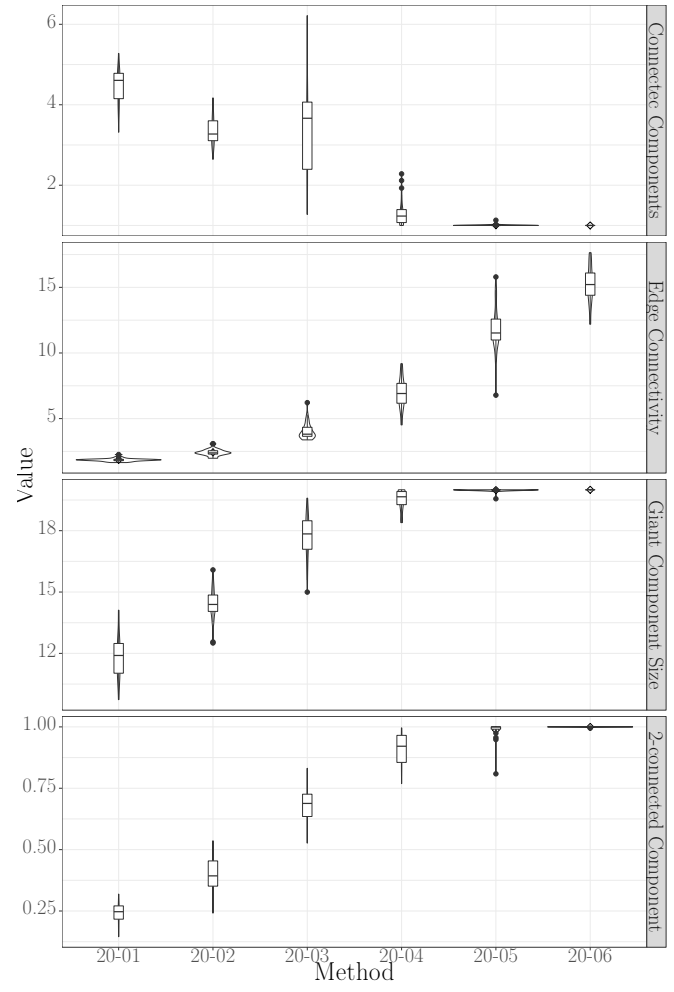

Fig. 3. Connectivity metrics for 20 UAVs.

simulation, we perform a linear regression $(a \times t+b)$ using the last 3500 steps.

c) Recent coverage: This metric introduced in [4] represents the percentage of coverage during the last 100 iterations. These 100 steps correspond to the pheromones' evaporation time. We exclude the 100 first iterations of our simulation to compute the mean value of the recent coverage.

d) Giant component: The giant component of a network is the connected subgraph (component) with the largest number of nodes in a network. We measure the giant component size of a swarm to gain insight about the size and variation of the largest connected component of the moving UAV swarm. In a realistic scenario, UAVs will disconnect from the swarm core due to obstacles avoidance or other spontaneous maneuvers. This means that disconnections resulting in isolated UAVs can occur.

e) Number of components: A component is defined as an isolated (or disconnected) subgraph of the considered network. Our approach can result in disconnections of not only isolated UAVs but groups of UAV. Measuring the number of groups (also components) during the lifetime of the network does complement the understanding of the networks' dynamic behavior already given by the giant component.

f) k-edge-connectivity for the giant component: A network has connectivity $k$ (or $k$-connectivity) if $k$ is the size of the smallest subset of nodes such that the network becomes disconnected if the subset is deleted. We only calculate the 2-connectivity of the giant component because the frequent isolated nodes or components of the UAV swarm would have a negative and unreasonable influence of the $k$-connectivity 


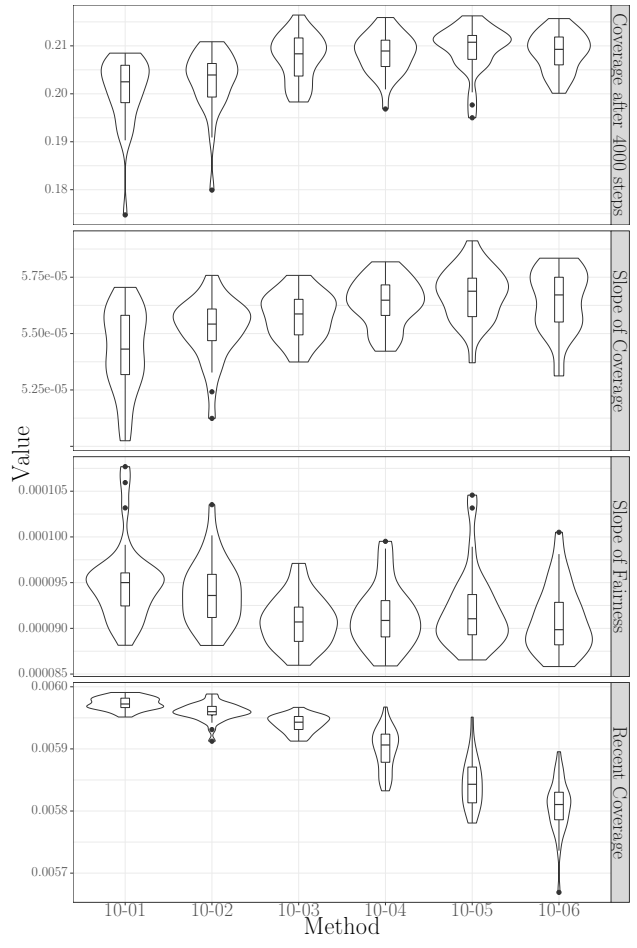

Fig. 4. Coverage metrics for 10 UAVs.

measure. Thus, if the giant component is 2-connected we note 1 , else we note 0 . At the end of the simulation, we compute the average value of this metric to obtain the percentage of the simulation time when the giant component is 2-connected.

g) Edge-connectivity: The purpose of this metrics is to evaluate how many edges can be removed to prevent this component to be disconnected. This is useful to evaluate how the swarm is resilient to communication issues. It comes as a complementary measure of the 2-connectivity to highlight the communication quality of the swarm.

\section{RESULTS}

The results obtained show the influence of the number of UAVs on the coverage and connectivity quality of the swarm. Summarizing the results, we produced a metrics-comparison chart which shows the correlation between specific metrics to each other. We discuss the connectivity metrics and afterward the coverage metrics. Figures 2, 3, 4 and 5 show the box plot of the data for all the measured metrics and a smooth density estimation.

\section{A. Connectivity metrics}

We first describe the results contained in Fig. 2 and Fig. 3. Concerning the connected components, the experiments underline that for force equal to $0.4,0.5$, and 0.6 , the number of connected components is very low: lower than two connected components for 10 and 20 UAVs. For these forces and 20 UAVs, the average value is close to one which means that the UAVs mainly remain connected. This is confirmed by the giant component metrics because the size of the giant component is in average equal to the number of UAVs for forces equal to $0.4,0.5$ and 0.6 for $20 \mathrm{UAV}$ s. However, it is only the case

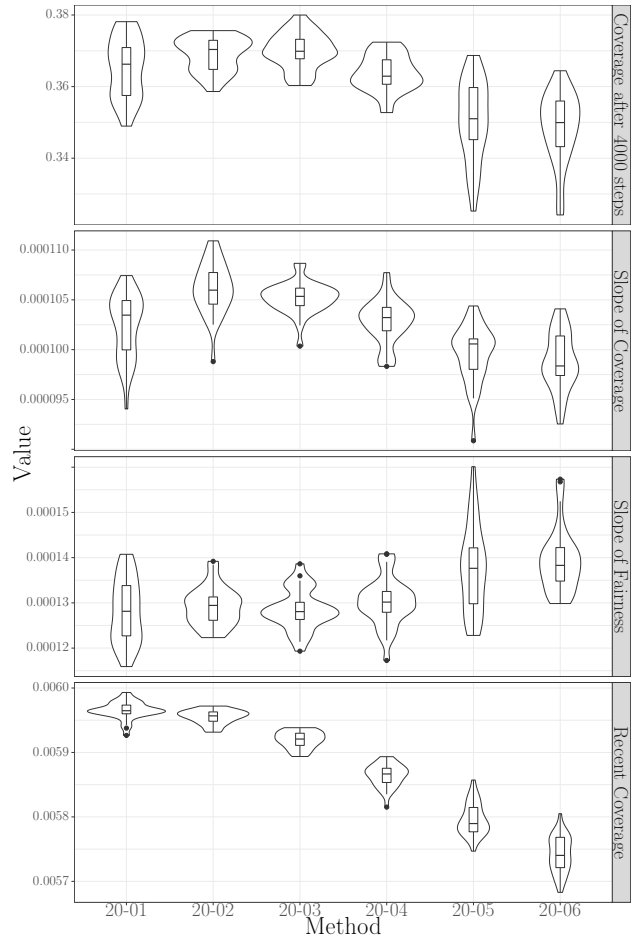

Fig. 5. Coverage metrics for 20 UAVs.

for forces equal to 0.5 and 0.6 for 10 UAVs. On the other hand, when the force is equal to 0.1, the number of UAVs in the giant component corresponds to about $50 \%$ of the total number of UAVs deployed.

After discussing the size of the giant component, we will use the two last metrics to analyze its properties. First, the 2-connected components are inversely proportional to the number of connected components with a smaller standard deviation for the low forces $(0.1$ and 0.2$)$. For forces equal to 0.5 and 0.6 , we observe that the values are close to 1 . It means that almost $100 \%$ of the time, the swarm is two-connected. Apparently, the value is similar for 10 or 20 UAVs. The edge connectivity of the giant component indicates how many communication links can be removed to maintain connectivity between the nodes of the components. This value remains very low for low force values: 0.1 and 0.2 and then it increases smoothly up to the $75 \%$ of the total number of UAVs for both (20 UAVs and $10 \mathrm{UAVs}$ ). For forces equal to 0.5 and 0.6 the edge connectivity of the giant component is higher than half of the number of UAVs. This means that our mobility model ensures a very resilient communication network when the force is above 0.4 .

\section{B. Coverage metrics}

In this subsection, we analyze the coverage metrics depicted by Fig. 4 and Fig. 5. We first consider the percentage of total area covered after 4000 steps for an optimal model. In this theoretical case, we consider that for each step the UAV covers a not already visited cell of the area. Thus, for 10 UAVs, the optimal value for this metric is $25 \%$ of the total area $(10 \times$ $4000 /(400 \times 400)=0.25)$. Thus, the average values of the results obtained here for $10 \mathrm{UAV}$ are in between $20 \%$ and 
$21 \%$ to the total coverage. On top of that, the force value has no impact on this metric. However, if we double the number of UAVs to 20 , the optimal value is $50 \%$. The average percentage result is around $37 \%$ for forces lower than 0.5 but it decreases to $35 \%$ when the force is stronger. The increase of UAVs does not seem to be efficient considering only this metric. From the value collected on the coverage ratio, we also compute the slope of coverage. Thus, there is a strong relation between the slope of coverage and coverage after 4000 steps. For this metric we can underline that for $20 \mathrm{UAVs}$, the best results are for forces equals to 0.2 and 0.3 .

The recent coverage indicates how the area are recently visited (during 100 steps) by the UAVs. If we consider 10 UAVs, the optimal value is the following one: 10 UAVs for 100 steps are divided by the total number of covered area: $10 \times 100 /(400 \times 400)=0.00625$. For a force equal to 0.1 the value is close to this optimal value, and it decreases slowly for the force 0.2 and 0.3 . Then it decreases faster for the other forces. For $20 \mathrm{UAVs}$, the results are very similar to the previous one. The expected optimal value for recent coverage is $20 \times 100 /(400 \times 400)=0.0125$ and the results are below 0.006 . This means that the increase in the number of UAVs is not sufficient to reach better recent coverage regarding the optimal value of the corresponding number of UAVs. This result is confirmed by the fairness metrics for $20 \mathrm{UAVs}$ where the fairness slope is higher for forces equal to 0.5 and 0.6. However, for $10 \mathrm{UAVs}$ this fairness slope is almost the same.

\section{Synthesis between coverage and connectivity metrics}

Based on the previously presented results, our approach exhibits superior performance for $10 \mathrm{UAVs}$ regarding recent coverage but does not produce similar results for 20 UAVs. Also, the best performing models on this from a coverage point of view are the models with forces equal to 0.1 and 0.2 . We explain this phenomenon by the low force that might generate numerous components where UAVs come back on already visited areas before the evaporation time of the pheromones exceeds. As the pheromones are repulsive, they prevent UAVs to reach an already visited area. Conversely, as the force is strong, the UAVs remain connected and overlap their covered area. This behavior is confirmed by the connectivity metrics where the size of the giant component is almost equal to half of the number of UAVs while the average number of components is two. This splitting into two swarms is something that frequently happens when "boids" avoid obstacles.

The results also showed that the values for 20 UAVs do not reproduce the results for 10 UAVs (regarding their respective optimal values). This implies that although we can expect to have significant benefits in using a UAV swarm with a higher number of UAVs, beyond a certain threshold it does not further improve the overall performance.

\section{CONCLUSIONS}

In this paper, we introduced the $\mathrm{CACOC}^{2}$ mobility model for UAV swarms. CACOC ${ }^{2}$ combines CACOC and a flocking model based on Reynolds "boids" where a flocking force is added to each CACOC direction choice. Our experimental results have permitted to calibrate the flocking force to have a mobility model combining coverage and connectivity constraints. Thus we have empirically defined the parameterization to obtain a mobility model efficient with 10 or 20 UAVs to cover a wide area with connectivity constraints. This parameter $f$ permits to equilibrate the swarm behavior with acceptable coverage performances. In future works we plan to introduce $\mathrm{CACOC}^{2}$ in a realistic UAV simulator to introduce the collision avoidance necessary for UAVs.

\section{ACKNOWLEDGMENTS}

The experiments in this paper were using the HPC facilities of the University of Luxembourg [17] - see http://hpc.uni.lu.

\section{REFERENCES}

[1] S. Hauert, S. Leven, J.-C. Zufferey, and D. Floreano, "Communicationbased leashing of real flying robots," in Proc. of IEEE International Conference on Robotics and Automation (ICRA), 2010.

[2] M. R. Brust, M. I. Akbas, and D. Turgut, "VBCA: A virtual forces clustering algorithm for autonomous aerial drone systems," in Proc. of IEEE Systems Conference (SysCon), 2016.

[3] M. Rosalie, G. Danoy, S. Chaumette, and P. Bouvry, "From random process to chaotic behavior in swarms of UAVs," in Proc. of ACM Symposium on Development and Analysis of Intelligent Vehicular Networks and Applications (DIVANet), 2016.

[4] G. Danoy, M. R. Brust, and P. Bouvry, "Connectivity stability in autonomous multi-level UAV swarms for wide area monitoring," in Proc. of ACM Symposium on Development and Analysis of Intelligent Vehicular Networks and Applications (DIVANet), 2015.

[5] M.-A. Messous, S.-M. Senouci, and H. Sedjelmaci, "Network connectivity and area coverage for UAV fleet mobility model with energy constraint," in Proc. of IEEE Wireless Communications and Networking Conference (WCNC), 2016.

[6] E. Yanmaz, "Connectivity versus area coverage in unmanned aerial vehicle networks," in Proc. of IEEE International Conference on Communications (ICC), 2012.

[7] S. Hauert, L. Winkler, J.-C. Zufferey, and D. Floreano, "Ant-based swarming with positionless micro air vehicles for communication relay," Swarm Intelligence, vol. 2, no. 2, pp. 167-188, 2008.

[8] E. Yanmaz and C. Bettstetter, "Area coverage with unmanned vehicles: A belief-based approach," in Proc. of IEEE Vehicular Technology Conference (VTC), 2010

[9] E. Yanmaz, C. Costanzo, C. Bettstetter, and W. Elmenreich, "A discrete stochastic process for coverage analysis of autonomous UAV networks," in Proc. of IEEE Globecom Workshops (GC Wkshps), 2010.

[10] M. Dorigo, M. Birattari, and T. Stutzle, "Ant colony optimization," IEEE Computational Intelligence Magazine, vol. 1, no. 4, pp. 28-39, 2006

[11] O. E. Rössler, "An equation for continuous chaos," Physics Letters A, vol. 57, no. 5, pp. 397-398, 1976.

[12] M. Rosalie, "Templates and subtemplates of Rössler attractors from a bifurcation diagram," Journal of Physics A: Mathematical and Theoretical, vol. 49, no. 31, p. 315101, 2016.

[13] C. W. Reynolds, "Flocks, herds and schools: A distributed behavioral model," SIGGRAPH Comput. Graph., vol. 21, no. 4, pp. 25-34, 1987.

[14] M. R. Brust and B. M. Strimbu, "A networked swarm model for uav deployment in the assessment of forest environments," in Proc. of IEEE International Conference on Intelligent Sensors, Sensor Networks and Information Processing (ISSNIP), 2015.

[15] E. Kuiper and S. Nadjm-Tehrani, "Mobility models for UAV group reconnaissance applications," in Proc. of IEEE International Conference on Wireless and Mobile Communications (ICWMC), 2006.

[16] J. Schleich, A. Panchapakesan, G. Danoy, and P. Bouvry, "UAV fleet area coverage with network connectivity constraint," in Proc. of ACM International Symposium on Mobility Management and Wireless Access (MobiWac), 2013.

[17] S. Varrette, P. Bouvry, H. Cartiaux, and F. Georgatos, "Management of an academic HPC cluster: The UL experience," in Proc. of IEEE International Conference on High Performance Computing \& Simulation (HPCS), 2014. 